\title{
Differences and Deficits in Psychological Research in Historical Perspective: A Commentary on the Special Section
}

\author{
Michael Cole \\ University of California, San Diego
}

\begin{abstract}
This commentary traces discussions of psychological differences and deficits from the mid-1950s to the current day, positioning the disciplinary discussions in the social-historical context in which they took place. The challenges of assessing diagnoses of deficit and the potential harms that result when misdiagnosis is implemented as social policy pervade the discussion over time.
\end{abstract}

Keywords: academic achievement, cultural deprivation, culture of poverty, nonrepresentative norms, psychological deficit

I have been invited to comment on the articles in this special section by virtue of the fact that I began several decades ago to study psychological tests associated with cultural, ethnic, and social class variations in psychological development (Cole \& Bruner, 1971; Cole, Gay, Glick, \& Sharp, 1971). The question of how differences in psychological test performance come to be interpreted as deficits was central to that line of inquiry. I take it to be my task to bring a historical dimension to the topic of psychological differences and deficits by contrasting how the issues were conceived "back in the day" with how they are conceived of at present.

As the first date for a "then and now" comparison, I have chosen the debates leading up to 1971 as the starting point. The current set of articles will provide evidence about the current scene (Summer 2012).

\section{Comparing Social and Historical Contexts: Then and Now}

Because we are dealing with issues that clearly arose in highly charged and tumultuous times, I will begin by sketching the larger social and historical circumstances within which psychologists were-and still are-conducting their research on the differencedeficit issue, before turning to the psychological literature itself. My account should of necessity be treated as a "thumbnail," not a full-blown picture.

\section{Then}

A useful starting date for my historical comparison is 1954, the year that the U.S. Supreme Court handed down the Brown v. Board of Education decision to end racial segregation in schools. What-

I wish to thank my colleagues at the Laboratory of Comparative Human Cognition (LCHC) for their critical reading of an early draft of this article and their generous suggestions for its improvement. The flaws I claim as my own.

Correspondence concerning this article should be addressed to Michael Cole, Laboratory of Comparative Human Cognition, University of California, San Diego, La Jolla, CA 92093. E-mail: mcole@ucsd.edu ever the flaws in subsequent execution of the will of the Court, the movement toward racial desegregation changed the developmental trajectories of millions of children by sending them to integrated schools. But it did not take long before people began to discover that no-longer-separate did not mean no-longer-unequal. The inequalities of the prior decade may have been displaced into formally integrated schools, but they did not disappear. Instead the problem was replaced by "the achievement gap." Poor children, and particularly poor children of color, continued to lag 30\%-40\% behind their middle class, largely European American neighbors in academic achievement. Something more was needed to achieve the hoped-for benefits of desegregation, because the sources of inequality went well beyond the educational system into the economy and social system as a whole.

That "something more" was the War on Poverty. When this war was declared in 1964, both the public at large and developmental psychologists in particular assumed (and probably still do assume) that intervention into developmentally dangerous trajectories should begin as early as possible. If academic underachievement is the danger, then providing a "head start" for those at the bottom of the scale should provide the necessary immunization. That consensus gave rise to Project Head Start as one means of ending poverty through education.

Head Start, like Brown v. Board of Education, was initiated to change developmental trajectories, and each of them involved the use of psychological tests and their prescribed modes of interpretation as their data of choice. Five years into its implementation, the data from Head Start were equivocal at best. The success or failure of this project was hotly debated in journals and by the popular press all throughout the later 1960's.

It should also be kept in mind that the summers following the initiation of the War on Poverty were hot in another, nonacademic, sense. It seemed that no sooner had war been declared than major domestic violence broke out: The Watts Riots (1965), then the Detroit and Newark Riots (1967). The murder of Martin Luther King Jr., who was supporting striking garbage workers at the time he was killed, brought together the issues of ethnicity and income in a particularly vivid way. The War on Poverty was not going as planned. 
Then there was that other war that intersected the War on Poverty - the war in Vietnam. It, too, was failing to go according to plan. Vietnam was heating up and with it the level of rejection of existing social institutions in the United States. Resistance to the war blended with the civil rights movement. In May of 1970 the domestic tension snapped: Four college students at Kent State University were killed by national guardsmen. The international war had become an internecine war.

This is no more than a rough sketch of the social-historical context "pre-1971." It was clearly a highly contentious and volatile time. Issues of diversity, income, education, and ethnicity were at the nexus of the contention. Psychologists found themselves right in the middle of the debate, as theorists, practitioners, and policy makers.

\section{Now}

When we consider the current global situation it appears both that a whole lot has changed and yet the core issues of ethnicity and social class inequality remain clearly visible. At a global level, the Cold War has ended. That was a war the United States could claim to have won. In its place we have "globalization," which has enormously increased international interaction and where issues of haves and have nots are now being fought out. And, unfortunately, the Cold War has been replaced by a myriad of local/regional conflicts and by a religious ideological holy war that has proven to be anything but cold. Reorganized and increased rates of international interaction have induced both economic development and a "War on Terror."

Overall economic inequalities within the United States have grown over recent decades. What economists refer to as "the great convergence" of income levels that preceded World War II was coming to an end by 1970 . In its place there have now been 40 years of a "great divergence," resulting in contemporary rhetoric and political conflict about "the $1 \%$ versus the $99 \%$ " and accusations of "class warfare" that have not been heard in this country since the 1930's. Internationally the degree of inequality is almost literally unimaginable, fueled directly by the triumph of EuroAmerican-Japanese capitalist democracies.

When we turn to contemporary thinking about social differences associated with demographic categories such as "poor" or "member of an ethnic minority," the situation has become amazingly more complex and slippery. On the one hand there is diversity of ethnicities due to events at the cultural-social-historical level, which I sketched out above. Owing to the wars of earlier decades and to economic inequalities at the global level, there has been a gigantic population shift in the United States. Socioeconomic class, ethnic, and linguistic diversity have increased rapidly to the point where those of European descent are expected to become a minority during my children's lifetimes. Simultaneously, fueled by the social forces unleashed by the civil rights movement, there have also been marked changes in the nature of gender relations, notions of disability, and social relations across hitherto repressed forms of diversity of all kinds. These changes have become a part of the fabric of American life, as reflected in popular culture. Whereas Ozzie and Harriet with its all-White, straight, two parent-two child family was the iconic image of family life in 1971, today Modern Family with its jumble of genders, ages, and ethnicities is the most popular American television program. ${ }^{1}$

\section{Psychological Theories of Difference and Deficit: Then and Now}

When we consider all of the ways in which contemporary American diversity and difference are experienced by children and adults alike, in law and in daily practice, it is well worth revisiting the questions of how psychologists should understand the relationship between difference and deficit, and the role we should play in shaping children's developmental trajectories through our judgments. Once again, it is helpful to compare then with now.

\section{Then}

The academic arguments about diversity and deficit at the end of the 1960s when Bruner and I undertook to write on the topic were polarized around explanations for the continuing achievement gap between middle class and working class children, with particular concern focused on African Americans.

Most of those who adopted a deficit view assumed that growing up in conditions of poverty deprives children of essential cultural conditions for normal intellectual development. ${ }^{2}$ In the parlance of the day, the "cultural deprivation" that accompanied poverty and ethnic marginalization produced a "deficit in psychological functioning." These cultural deficiencies included less mutuality in social interaction, less emphasis on reasoning and means/end relationships in maternal instruction, reliance on punishment and negative reinforcement, engagement in less strategic forms of play, reliance on immediate and concrete forms of reward for learning, lack of impulse control, and the like.

This line of thinking tended, in the heat of the debate, to find some extreme expressions. For example, J. McVicker Hunt, influential author of Intelligence and Experience (1961, p. 323), suggested that "The difference between the culturally deprived and the culturally privileged is, for children, analogous to the difference between cage reared and pet-reared rats and dogs." Others suggested that the children in poverty grew up in homes where there was too much stimulation of the wrong kind. Cynthia Deutsch, an early advocate of this perspective characterized the situation as follows:

The slum child is more likely than the middle-class child to live in a crowded, cluttered home- but not cluttered with objects that can be playthings for him. ... [I]n the terms used earlier, the slum child has, in his stimulus field, both less redundancy and less education of his attention to the relevant properties of stimuli. As a result, he could be expected to come to school with poorer discrimination performance than his middle-class counterpart. (Deutsch, 1968, p. 79)

Special emphasis was placed upon culturally linked deficiencies in language and its use; the poor were said not only to use language less, but to be restricted to speaking a less elaborated form of language that failed to support intellectual development. This view underpinned the work of Carl Bereiter and Siegfried Engelmann, work that drew special attention in part because it was explicitly

\footnotetext{
${ }^{1}$ It should be noted that one social feature that did not change was the solid economic well-being of characters in these shows. The dream, if not the reality, of the idea of economic equality endured.

2 The obvious exception was Arthur Jensen's (1969) claim that the achievement gap reflects a genetic deficit.
} 
linked to a strategy for compensatory education that was compatible with the environmental learning theories of the day. I quote at length because the passages give an excellent feel for the underlying assumptions and their connection to a theory of compensatory early education:

The speech of the severely deprived children seems to consist not of distinct words, as does the speech of middle-class children of the same age, but rather of whole phrases or sentences that function like giant words. That is to say, these "giant word" units cannot be taken apart by the child and re-combined; they cannot be transformed from statements to questions, from imperatives to declaratives, and so on. Instead of saying "He's a big dog," the deprived child says "He bih daw." Instead of saying "I ain't got no juice," he says "Uai-ga-na-ju." Instead of saying "That is a red truck," he says "Da-re-truh." Once the listener has become accustomed to this style of speech, he may begin to hear it as if all the sounds were there, and may get the impression that he is hearing articles when in fact there is only a pause where the article should be. He may believe that the child is using words like it, is, if, and in, when in fact he is using the same sound for all of them-something on the order of "ih." (This becomes apparent if the child is asked to repeat the statement "It is in the box." After a few attempts in which he becomes confused as to the number of "ih's" to insert, the child is likely to be reduced to a stammer.) (Bereiter \& Engelmann, 1966, pp. 34-35)

To deal with this presumed absence of language, Bereiter and Engelmann focused on teaching a "language of thought" as the gateway to instruction and education. Crediting Engelmann for the idea, Bereiter described the logic of their remedial approach in the following terms:

As Engelmann saw it, the child's primary need was for a language that would enable him to be taught. Once the child had that, you could go on and teach him anything else you pleased. Such a language did not have to be distilled from a recording of actual verbal behavior but could be constructed, much as Basic English was constructed, by a consideration of the needs it had to serve. Such a language could be taught to children in a relatively short time (in practice, two to six months), and it would then be possible to add the refinements of complete English and also to teach other things in a more direct and normal manner.

Teaching disadvantaged children a miniature language that someone else has made up for them may sound a bit 1984ish to the doubters among us; but realize that it is regular English, just a stripped-down version of it, and that the principle of starting with a miniature system which is part of, but more easily grasped than, the entire system is a respectable and widely used pedagogical device. Methods of reading instruction that begin with a limited vocabulary that follows a few consistent spelling rules are an example, as are physics lessons that begin with consideration of a homogeneous frictionless environment. (Bereiter, 1968, p. 341)

Whether the image is one of children-as-rats isolated in their cages or children living in situations of uninterpretable chaos, the solution was the same: provide children from as early an age as possible with the necessary cultural-environmental experiences.

\section{Cultural Difference, Not Cognitive Deficit}

It should come as no surprise that these ideas met with heated opposition from linguists with extensive experience working in the African American community, including William Labov, a linguist who studied social class and ethnic/racial variations in language use. Labov argued that the low scores on standardized language competence tests of children who speak nonstandard or African American English (AAE) arise from the inadequacies of the testing, not the inadequacies of the children. Such children come to school speaking a distinctive dialect of English (Labov, 1970, 2010).

To demonstrate his point Labov arranged for a comparison of the language used by an 8-year-old boy, in three settings: (a) the Illinois Test of Psycholinguistic Abilities (a common object is placed on the table in front of the child, who is asked, "Tell me everything you can about this"); (b) a visit from a local Black researcher to the child at home in which the topics were chosen to be of direct interest to the child, such as gangs and street fighting; and (c) a visit from the same researcher who this time arranged for the child's friend to be present, brought along snacks, and started up a conversation about insults directed at mothers, a taboo subject but also the topic of well-known varieties of verbal duels (sounding) in the community.

The test situation engendered a painful sequence of verbal prods from the interviewer and minimal verbal productions from the child, with pauses of up to 20 seconds between questions and monosyllabic answers. The standard deficit interpretation of this behavior had it that the child had failed to acquire grammatical competence; Labov's interpretation was that the child was actively attempting to avoid saying anything in a situation where whatever he says could literally be held against him (Labov, 1970).

This interpretation appeared to be disconfirmed when the same result ensued in the presumably less formal and locally more intriguing discussion at home about street fighting. It is only the third situation, which created an almost party-like situation, that produced dramatic changes in the child's speech. Not only did the child go well beyond one-word replies to questions, but he actively competed for the floor and talked excitedly with both his friend and the researcher, demonstrating that he was indeed linguistically competent, even if the language was not standard English.

Labov's findings epitomized the position of the "difference" advocates. From this perspective, test-like, one on one, adult-child interactions grossly underestimated the child's verbal abilities. Second, the social situation, including the way the adult used language, appeared to be an essential determinant of the child's verbal behavior and manifest intellectual competence.

Bruner and I abstracted several conclusions from this and related research:

(a) Formal experimental equivalence of operations does not insure de facto equivalence of experimental treatments; (b) different subcultural groups are predisposed to interpret the experimental stimuli (situations) differently; (c) different subcultural groups are motivated by different concerns relevant to the experimental task; (d) in view of the inadequacies of experimentation, inferences about lack of competence among black children are unwarranted. (Cole \& Bruner, 1971, p. 869)

Note that these conclusions did not explain the low levels of test performance and the high levels of school failure among poor and marginalized American children. As we put it at the time,

While it is very proper to criticize the logic of assuming that poor performance implies lack of competence, the contention that poor performance is of no relevance to a theory of cognitive development 
and to a theory of cultural differences in cognitive development also seems an oversimplification. (Cole \& Bruner, 1971, p. 871)

After reviewing a good deal of the literature on factors associated with social class, ethnic, and cultural variations in cognitive performance under a wide range of testing procedures, we reached a conclusion that provides a baseline for judging the extent to which the intervening 40 years have brought about progress in our understanding of the relation between cognitive differences and cognitive deficits:

\begin{abstract}
The problem is to identify the range of capacities readily manifested in different groups and then to inquire whether the range is adequate to the individual's needs in various cultural settings. From this point of view, cultural deprivation represents a special case of cultural difference that arises when an individual is faced with demands to perform in a manner inconsistent with his past (cultural) experience. In the present social context of the United States, the great power of the middle class has rendered differences into deficits because middleclass behavior is the yardstick of success. (Cole \& Bruner, 1971, p. 874)
\end{abstract}

\section{Difference and Deficit Now: The Articles at Hand}

The first things one notes immediately on reading the articles in this special section of Developmental Psychology are that developmentalists continue to wrestle with the issues Bruner and I addressed in 1971, and that the range of human diversity being systematically studied has widened as part of the secular trends toward acceptance of various kinds of diversity that were once either ignored or treated as pathologies. Issues of socioeconomic/ ethnic diversity are the focus of half the articles, while the remainder address issues where the biological state of the child is known to be compromised in some way.

Beginning with the domain with which I am most familiar, that of ethnicity and social class, these articles show clear continuities and discontinuities with the debates of 1971 . With respect to continuities, the article by Hoff (2013) provides a myriad of evidence gathered by her and many others in support of the view that poor children are exposed to language that is more restricted in amount, variety, and complexity than middle class children. Moreover, the child-directed language of poor mothers is used more frequently for purposes of directing children's behavior than for purposes of "eliciting and maintaining conversation." These children perform relatively poorly on various measures of oral language development and subsequently are slower to acquire literacy and perform more poorly in school. These results mirror well the then-available evidence that motivated the deficit view of the 1960s and 1970s.

However, Hoff (2013) is well versed in the lessons taught by Labov and a variety of other scholars: These same children may be highly skilled in language practices about which their middle class peers are clueless. Moreover, skill in local, specialized uses of language may even predict school failure. From Hoff's point of view, given the enormous importance of education to economic well-being, the observed language differences are, de facto, culturally organized academic (life income) deficits. Hoff's conclusion is that, pragmatically speaking, the evidence calls for society to provide extra support for the development of English skills, support that begins early whether the child is African American or comes from (say) a Spanish-speaking home and neighborhood.
Pearson, Conner, and Jackson (2013) focus more than does Hoff on the evidence that AAE is a complex, rule-governed, perfectly normal linguistic system that overlaps, but is not coextensive with, general American English (GAE). They note that while professional associations may argue for treating knowledge of AAE as a "communicative asset" for learning, in popular culture (including the popular culture of many teachers and admired African American leaders such as Toni Morrison and Bill Cosby, who found use of AAE "garbage") it is considered a "plague" that carries a strong social stigma.

Instead of looking to unspecified sources of support for learning GAE during infancy and early childhood, Pearson and colleagues look to better educated teachers and a supportive school system where teachers can distinguish dialect from disorder. Teachers need to be able to provide "dialect-sensitive" and "culturally sensitive" instruction that promotes awareness and appreciation of dialect variation so that children are properly supported to acquire skills essential to the ability to read, such as phonemic awareness. In addition, the instructional circumstances should be such that the social stigma of speaking a dialect that converts difference into deficit is replaced by an appreciation of linguistic and cultural diversity that creates effective bilingual (bidialectical) and bicultural development.

Fryberg et al. (2013) provide a different approach to sources of poor school achievement. They focus not on language but on cultural identity and cultural differences in the interpretation of assertive student behavior. They report the intriguing finding that First Nations Canadian youth who self-reported strong identification with either their home culture or what they refer to as the mainstream White culture tended to have higher academic performance. The youth who fail to identify strongly with either culture are those at particular risk for academic failure ... unless (contrary to their home cultural norms) they are perceived as "assertive." (Assertiveness was indexed by teacher ratings of such behaviors as defending one's own view in class, participating willingly in class discussion, and questioning rules that seem unfair or unclear.)

This study provides provocative evidence of significant heterogeneity in school attainment among a culturally marginalized group, a finding that clearly points to a "difference" interpretation of cultural variations in academic performance not focused on questions of language development. Correlations among selfreports do not permit causal attributions concerning either home background or schooling practices, but the authors are probably justified in concluding that fostering academic achievement among First Nations children may "involve legitimating a variety of ways of being."

The remaining articles in this collection, where issues of difference and deficit are linked to biological causes, provide instructive cases to help us think about how ideas of deficit and difference in developmental comparisons have changed over the past 40 years. The case of language development among children who experience severe hearing loss, for example, highlights the intimate relation between culture and language development in a new way that is signaled by the distinction between the terms "deaf" and "Deaf." The case of autism and the evolution of notions of neurodiversity raise these same issues involving a different mixture of cultural and biological factors.

In 1971 there was still great uncertainty about the status of American Sign Language: Was it a crude system of largely iconic 
gestures or a bona fide language with its own forms of phonology, morphology, syntax, and so on? "Deaf and dumb" referred not only to hearing loss and lack of vocalization but was often interpreted by hearing people as the rough equivalent of "stupid." By 1971, scholars were beginning to understand that ASL is a fully developed human language (Stokoe, 1960/2005), although this idea was not yet widely accepted. In 1972, James Woodward proposed a distinction between the inability to hear (deafness) and the ability to use ASL as a member of a community with its own customs, beliefs, and values, which he designated as Deafness. But broad acceptance of these ideas was slow in coming. As late as the early 1980s I was told by a well-known senior colleague that it was out of the question to hire a deaf scholar with a $\mathrm{PhD}$ as a faculty member because it would be impossible for the person to lecture and interact with students. Today, ASL and other sign languages originating in many different societies are accepted as fully developed human languages. Moreover, it is recognized that Deaf culture is a vibrant and dynamic medium for sign language development, and Deaf scholarship is widely accepted (Padden \& Humphries, 1983, 2005).

Whether or not serious hearing loss represents a deficit has been shown to depend critically upon circumstances. Children born into a community where sign language is prevalent acquire that language. Consequently, as Lederberg, Schick, and Spencer (2013) put it, they are on an "equal but different trajectory." However, insofar as such children must deal with a dominant social environment in which sign language is absent and their hearing loss produces communicational difficulties, a deficit will generally develop in such circumstances. These are just the kinds of condition that Bruner and I identified as those that convert differences into deficits. Also serious is the case of children born into hearing homes where ASL is unknown or perhaps disapproved of because of problems that parents see lying ahead for their children. As Lederberg et al. point out, children in such a situation experience delayed language acquisition and may never achieve normal adult levels in either signed or oral language.

Even children whose ASL development is perfectly normal can experience severe difficulties acquiring literacy, owing to the difficult task of mapping ASL phonemes produced through patterns of visually perceived movement with phonemes based upon sound patterns to which the learner has little or no access. In a society where literacy is the gateway to education, this problem poses ongoing challenges for those affected.

The advent of what Lederberg et al. (2013) refer to as advanced audiological interventions raises new and difficult issues concerning difference and deficit that have yet to garner significant attention outside the community of Deaf and deaf people directly concerned. Quite apart from the shortcomings of such devices, those who fought for the recognition of ASL as a language and the mandated social supports for inclusion that have made it possible for Deaf scholars to work in institutions dominated by hearing people face the loss of a culture that has sustained a valued community. Unsurprisingly, arguments over the value of cochlear implants and other technologies that seek medical solutions to hearing loss have given rise to acrimonious controversy over differences and deficits in the Deaf and deaf communities. The full inclusion of people with severe hearing loss is now demonstrably possible. But it remains an open question just how much hearing people will support such accommodations and how successful the research community will be in finding ways to support the acquisition of literacy that is central to social and economic well-being in the modern world.

Just as our understanding of ASL and our acceptance of the reality of a Deaf community have changed dramatically over the last 40 years, conceptions of autism have morphed substantially. Forty years ago autism was presumed to be a unitary biological deficit in which social impairments, communication difficulties, and restricted, repetitive, and stereotyped patterns of behavior were combined with mental retardation. Today, autism is more widely understood as a spectrum of behaviors in which the supposedly clumped symptoms of what was seen as a single disorder are now viewed as variably present.

Each of the remaining two articles provides a window on new perspectives for thinking about difference and deficit with respect to what is now referred to as autism spectrum disorder (ASD). In the case of loss of hearing, knowledge of the specific biological causes is extensive and agreed upon. There are genetic markers for deafness and there is a well-developed medical theory of the various ways in which deafness can arise from disease and from environmentally induced brain lesions. By contrast, as Norbury and Sparks (2013) point out, while there is broad agreement that ASD is the result of a biological difference in brain function there is no consensus concerning specific biological markers or appropriate biological tests for ASD, so psychologists must depend upon behavioral data. Once one begins to rely on behavioral data for diagnosis and treatment, justification of difference/deficit interpretations is subject to the effects of culture in both the interpretation of the problem and the behavioral regimens that are intended to cure or ameliorate specific forms of behavior.

The picture that emerges from the two articles dealing with ASD provides rich evidence of how complex the issue of difference and deficit can become, as well as the identification of where children fall along "the spectrum." First of all, such classifications depend upon whose point of view is taken; it seems perfectly reasonable that parents whose child displays, say, what the doctors call Asperger's syndrome may feel quite certain their child is disabled and that Asperger's syndrome is a serious psychological deficit. Manifestation of the symptoms means at least reorganization of socially accepted life routines and an increase in having to deal with the medical community, which is no picnic.

At the same time, these children may display behaviors widely deemed by their social group to be talents and may be engaged with others in a manner that is distinctly social, although perhaps in a way that seems unconventional. From this perspective, the rise of what Kapp, Gillespie-Lynch, Sherman, and Hutman (2013) refer to as "autistic self-advocates" and of the neurodiversity movement is also perfectly reasonable. From this perspective it is society, not the individual, that is deficient.

The complexities of characterizing different points along the "autism spectrum" fully justify the title of the article by Kapp and his colleagues (2013): Are we dealing with clearly defined deficits, human variety (differences) that should be accepted and incorporated into social life, or both? Their answer, as I understand it, is both: autism spectrum disorder, especially at the more severe end of the spectrum, is interpreted as a deficit, the consequences of which should be ameliorated to best of society's ability. A clear change from 40 years ago is that the humanity of people who manifest ASD symptoms is currently acknowledged, and despite 
their marked differences from social norms their participation in everyday social life is, under some conditions at least, made possible.

Finally, when we consider the issue of neurodevelopmental disorders such as ASD in cross-cultural perspective, we appear to come full circle back to the ideas that so vexed psychologists and society in 1971. I confess to a feeling of "déjà vu all over again" when reading Norbury and Sparks's (2013) discussion relating problems of testing for ASD in other cultures. As their review admirably illustrates, many of the problems vexing developmentalists who wish to study ASD in other cultures mirror the difficulties of cross-cultural research more generally. For example:

- When Puerto Rican kids are shown a picture of a knife and say "for cutting" is it an indication of language delay or a preference for describing over labeling?

- What are we to make of the fact that ASD kids increase eye contact when they are interested in the topic at hand?

- What is the significance of the fact that parents and teachers often differ in their evaluation of ASD children's social communication/interaction behaviors?

- When Chinese children who are suspected of ASD fail to look peers in the eye is it really a symptom of ASD or of the inappropriateness of such behavior in Chinese culture?

These are just the kinds of questions that undermined confidence in the conclusions drawn from cross-cultural research on cognitive development all through the 1960s and later decades. They are the same questions to which Labov issued his challenge in 1970. And now they are being discovered all over again in research on neurodevelopmental disorders.

\section{Some Reflections on Difference and Deficit Then and Now}

A good deal has changed over the past 40 years in the study of differences and deficits in child development. No one today believes that the average poor African American child comes to school bereft of language. ASL is recognized as a fully developed language that supports a dynamic Deaf culture. The behavioral peculiarities of people referred to as autistic are no longer considered symptomatic of a single neurological abnormality and deficiency on a single path of development.

But some things have not changed. The achievement gap remains. Poverty has become the lot of many more families, bringing with it substandard education, nutrition, and health care. Failure to speak standard middle class English continues to stigmatize vast numbers of children, who disproportionately fail to complete secondary education and whose teachers may be in danger of losing their jobs if they resort to a child's home language in the service of instruction. The notion of a culture of poverty has reemerged as a powerful force guiding the education of the poor and marginalized (Payne, 2005). If anything, the presumed consequences of growing up in poverty are assessed as more dire now, owing to the surge of interest in the role of nutrition in prenatal development and data that warn about the bad effects of poverty on the brain (Hackman, Farah, \& Meaney, 2010).

With so many important topics on developmentalists' agenda, as reflected in the articles under discussion, it is difficult to comment on all the relevant issues. Of the many topics worth further reflection and stimulated by these articles, I will restrict myself to two which bear closely on each other: the insidious harm caused by using norms not derived from the group in question to arrive at judgments about their state of development, and the social challenges that arise when it is discovered that presumably immutable deficits attributed to individuals can be ameliorated or obliterated by changes in social practice.

\section{Beware of Nonrepresentative Norms}

The issue of using appropriate norms is particularly prominent in the articles by Hoff (2013) and Pearson et al. (2013), on language development and schooling, as well as the article by Norbury and Sparks (2013) on the problem of assessing the prevalence and presumed varieties of ASD in radically different cultural groups. I will focus on the language assessment issue because it is a topic with which I have more experience and for which there is currently more information. But the same issues arise with respect to any sort of psychodiagnosis based upon behavioral data that calls for socially organized interventions.

The problem of using inappropriate norms for judging language and cognitive development is made explicit by Erika Hoff (2013). She quite correctly notes that the assessment of language development needs to done cautiously when norms derived from middle class children are used to assess language development among poor children because such norms "may not tap the skills of children from other backgrounds" (Hoff, 2013, p. 7). Yet virtually all of the examples of socioeconomic status (SES) differences she provides are subject to this limitation, and her caution does not preclude her from generalizations based on such data such as "Grammatical development is also affected by SES" (Hoff, 2013, p. 5). Other than underestimating a valued ability, what difference does the resulting measurement error make?

Research by William Hall and his colleagues (Hall, Nagy, \& Linn, 1984), using unobtrusive auditory recordings in homes and classrooms, suggests an answer. They found, as the empirical research reviewed by Hoff (2013) would lead us to expect, that poor, Black children experience a more restricted range of vocabulary than their middle class, European American peers in the home. However, they also found that the vocabulary of the poor Black children was not simply a subset of the vocabulary of their peers. To be sure, roughly two thirds of the vocabulary sampled was common to all groups, but one sixth was specific to poor Black homes while the remainder was specific to middle class homes.

Hall et al. (1984) went on to examine the readability formulas derived from those vocabulary norms and used to design basal readers. What they found was that vocabulary unique to middle class homes was overrepresented and when used to calculate readability formulas for basal readers, they underestimate the difficulty of the texts for AAE speakers who are in effect required to learn to read using vocabulary that they are not familiar with. The reverse never happens. Based upon this analysis of basal readers, Hall and his colleagues noted that existing reading formula designers "unintentionally but effectively build class and race bias into their lists" (Hall et al., 1984, p. 478). As a consequence of this bias poor/Black children are confronted with a more difficult task in learning to read because they are learning with 
relatively unfamiliar vocabulary. They conclude, "If curricula are not changed, we must at least be aware that we are demanding much more of those children whose lives are not represented in the materials they use in school" (Hall et al., 1984, p. 479).

The problem is not restricted to basal readers. Hall and Tirre (1979) showed that the same problem appeared in several of the standardized tests of intellectual/academic ability, such as the Wechsler Intelligence Scale for Children (WISC) and the Stanford-Binet. Hall and Tirre did not pursue their findings, but more recently Fagan and Holland did (Fagan \& Holland, 2002, 2007). They showed that when African American and White subjects were presented with materials that were either generally accessible to both groups, or that depended upon specific knowledge (specific vocabulary items, or proverbs of the sort used in IQ tests), group differences appeared only for the items that were not common across the two groups, leading them to the conclusion that "exposure to information, rather than intellectual ability, may account for racial differences in IQ" (Fagan \& Holland, 2002, p. $385)$.

In like manner, a wide variety of experimental studies have shown that when local norms are used in constructing materials for use in the study of memory development, widely reported social class and ethnic differences in performance presumably indicative of a failure to develop and deploy conceptual abilities simply disappear (Hall, Reder, \& Cole, 1975; Orasanu, Lee, \& Scribner, 1979).

These findings point to one direction that developmental psychologists and education should take: Be certain that the methods you use to arrive at conclusions about difference and deficit do not inadvertently misdiagnose the problems that motivated your research in the first place. Misdiagnosis leads to ineffective treatment, treatment that may make the problem worse (Labov, 2003).

\section{Implementing Effective Policies: A Question of Social Values, Not Individual Responsibility}

Assuming that our research leads to clear evidence of the sources of psychological differences, and even that we can with some certainty specify changes in environmental circumstances to reduce those differences if they are considered harmful, we must still confront the fact that the conditions producing the problematic developmental trajectories are likely to be firmly embedded in social, economic, and legal practices that maintain the very conditions whose consequences we decry. It has long been known that if middle class parents are placed under even mild stress, they ignore conversational openings they might otherwise have attended to and begin to use the kind of controlling, directive language that is said to be the proximal cause of poor children's inadequate language development (Zussman, 1980). In poor households, high levels of stress are endemic, so such language use should be expected. It has also long been known that when parents work in conditions that offer little autonomy or individual initiative, parents recreate such conditions for their children at home (Kohn, 1977). The solution-end poverty and exploitation-is clearly beyond the control of psychologists, or political leaders, to arrange. The United States has lost the War on Poverty.

It seems natural, then, to focus on social institutions, such as the schools, to provide forms of instruction that, in effect, render children bicultural and bilingual. But to accomplish this feat re- quires not only teachers who are well educated in the issues and who are provided the required working conditions, but communities that cease to stigmatize children's home language and culture so that when teachers use methods shown to be successful, they are widely adopted. For example, if you are trying to teach children about sound (sign)-written symbol correspondences, it is almost certainly a good idea to use vocabulary with which the children are familiar and reading materials that motivate the children to learn more about matters of genuine interest to them. But this course of action is almost certain to bring you into conflict with parents who do not want their children attending schools with "those people." Nor should we ignore the fact that many poor parents who insist on obedience and respect in adult-child interactions are likely to object to middle-class style instruction, that "hidden curriculum" that middle class teachers are likely to use, a point made repeatedly by scholars such as Lisa Delpit (1993).

Analogous considerations apply in the cases involving some form of biological difference. The inclusion of Deaf people in a variety of social practices, even Deaf people who are highly educated, requires that social institutions make provisions for their full participation, such as the presence of translators in circumstances where communication ordinarily takes place in spoken language. The inclusion of movement-impaired people who must use a wheelchair presupposes a variety of modifications of the physical environment to provide physical access, and a cultural environment that treats impaired movement as a challenge for the community to overcome, not a personal problem for the individual and her/his family to deal with. Creating social environments where people with neurological differences can enrich their own lives and the lives of others through their participation also requires not only the provision of tangible social resources, but a cultural understanding that removes the stigma associated with the given condition, a stigma that serves as an excuse not only for bad science but for the devaluing of human life. Differences among human beings are essential to human life in the same way that biodiversity is essential to life on earth more generally. In the long run, if differences are also deficits in any fundamental way then those differences will disappear, only to give rise to new differences that in turn will create the social conditions for judgments about what is and is not a deficit. In Darwin's world, no less than the New Testament, we can expect that in the fullness of time, "the last will be first and the first will be last."

In the meantime, as developmental psychologists, we should be as careful as possible to be a part of the solution to humanity's problems and to avoid turning differences into deficits in the guise of promoting development.

\section{References}

Bereiter, C. (1968). A nonpsychological approach to early compensatory education. In M. Deutsch, I. Katz, \& A. R. Jensen (Eds.), Social class, race, and psychological development (pp. 337-346). New York, NY: Holt, Rinehart and Winston.

Bereiter, C., \& Engelmann, S. (1966). Teaching disadvantaged children in the preschool. Englewood Cliffs, NJ: Prentice-Hall.

Cole, M., \& Bruner, J. S. (1971). Cultural differences and inferences about psychological processes. American Psychologist, 26, 867-876. doi: 10.1037/h0032240

Cole, M., Gay, J., Glick, J. A., \& Sharp, D. (1971). The cultural context of learning and thinking. New York, NY: Basic Books. 
Delpit, L. D. (1993). The silenced dialogue: Power and pedagogy in educating other people's children. In L. Weis \& M. Fine (Eds.), Beyond silenced voices: Class, race, and gender in United States schools (pp. 119-139). Albany, NY: State University of New York Press.

Deutsch, C. P. (1968). Environment and perception. In M. Deutsch, I. Katz, \& A. R. Jensen (Eds.), Social class, race, and psychological development (pp. 58-85). New York, NY: Holt, Rinehart and Winston.

Fagan, J. F., \& Holland, C. R. (2002). Equal opportunity and racial differences in IQ. Intelligence, 30, 361-387. doi:10.1016/S01602896(02)00080-6

Fagan, J. F., \& Holland, C. R. (2007). Racial equality in intelligence: Predictions from a theory of intelligence as processing. Intelligence, 35, 319-334. doi:10.1016/j.intell.2006.08.009

Fryberg, S. A., Troop-Gordon, W., D’Arrisso, A., Flores, H., Ponizovskiy, V., Ranney, J. D., ... Burack, J. A. (2013). Cultural mismatch and the education of aboriginal youths: The interplay of cultural identities and teacher ratings. Developmental Psychology, 49, 72-79. doi:10.1037/ a0029056

Hackman, D. A., Farah, M. J., \& Meaney, M. J. (2010). Socioeconomic status and the brain: Mechanistic insights from human and animal research. Nature Reviews Neuroscience, 11, 651-659. doi:10.1038/ nrn2897

Hall, W. S., Nagy, W. E., \& Linn, R. L. (1984). Spoken words: Effects of situation and social group on oral word usage and frequency. Hillsdale, NJ: Erlbaum.

Hall, W. S., Reder, S., \& Cole, M. (1975). Story recall in young Black and White children: Effects of racial group membership, race of experimenter, and dialect. Developmental Psychology, 11, 628-634. doi: 10.1037/0012-1649.11.5.628

Hall, W. S., \& Tirre, W. C. (1979). The communicative environment of young children: Social class, ethnic, and situational differences. Champaign, IL: University of Illinois at Urbana-Champaign.

Hoff, E. (2013). Interpreting the early language trajectories of children from low-SES and language minority homes: Implications for closing achievement gaps. Developmental Psychology, 49, 4-14. doi:10.1037/ a0027238

Hunt, J. M. (1961). Intelligence and experience. New York, NY: Ronald Press.

Jensen, A. (1969). How much can we boost IQ and scholastic achievement? Harvard Educational Review, 39, 1-123.

Kapp, S. K., Gillespie-Lynch, K., Sherman, L. E., \& Hutman, T. (2013). Deficit, difference, or both? Autism and neurodiversity. Developmental Psychology, 49, 59-71. doi:10.1037/a0028353
Kohn, M. L. (1977). Class and conformity. Chicago, IL: University of Chicago Press.

Labov, W. (1970). The logical non-standard English. In F. Williams (Ed.), Language and poverty (pp. 153-190). Chicago, IL: Markham Press.

Labov, W. (2003). When ordinary children fail to read. Reading Research Quarterly, 38, 128-131.

Labov, W. (2010). Unendangered dialect, endangered people: The case of African American Vernacular English. Transforming Anthropology, 18, 15-27. doi:10.1111/j.1548-7466.2010.01066.x

Lederberg, A. R., Schick, B., \& Spencer, P. E. (2013). Language and literacy development of Deaf and hard-of-hearing children: Successes and challenges. Developmental Psychology, 49, 15-30. doi:10.1037/ a0029558

Norbury, C. F., \& Sparks, A. (2013). Difference or disorder? Cultural issues in understanding neurodevelopmental disorders. Developmental Psychology, 49, 45-58. doi:10.1037/a0027446

Orasanu, J., Lee, C., \& Scribner, S. (1979). The development of category organization and free recall: Ethnic and economic group comparisons. Child Development, 50, 1100-1109. doi:10.2307/1129337

Padden, C., \& Humphries, T. (1983). Deaf in America. Cambridge, MA: Harvard University Press.

Padden, C., \& Humphries, T. (2005). Inside Deaf culture. Cambridge, MA: Harvard University Press.

Payne, R. K. (2005). A framework for understanding poverty. Highlands, TX: Aha! Process.

Pearson, B. Z., Conner, T., \& Jackson, J. E. (2013). Removing obstacles for African American English-speaking children through greater understanding of language difference. Developmental Psychology, 49, 31-44. doi:10.1037/a0028248

Stokoe, W. C., Jr. (2005). Sign language structure: An outline of the visual communication systems of the American Deaf. Journal of Deaf Studies and Deaf Education, 10, 3-37. doi:10.1093/deafed/eni001 (Reprinted from Studies in linguistics, Occasional Papers 8, 1960, Department of Anthropology and Linguistics, University of Buffalo, Buffalo, NY)

Woodward, J. (1972). Implications for sociolinguistic research among the Deaf. Sign Language Studies, 1, 1-7.

Zussman, J. U. (1980). Situational determinants of parental behavior: Effects of competing cognitive activity. Child Development, 51, 792 800. doi: $10.2307 / 1129466$

Received June 20, 2012

Accepted June 25, 2012 